lymphatic system. Also, eosinopenia appeared in the cat ${ }^{11}$ following estrogen administration, while Bottlglioni and Sturani ${ }^{15}$ found that treatment with estrogens resulted in eosinophelia in man as well as in cattle ${ }^{16}$.

This action of estrogens upon the leucocytes has been explained by: a) a direct action on the haemopoietic organs either by stimulating the maturation of the cells in the bone marrow with a consequent release of leucocytes or by increasing the rate of release of mature leucocytes already in reserve ${ }^{17}$; b) an indirect action via the neurohormonal control of leucopoiesis particularly ACTH ${ }^{18}$.

The whole series of this work will stimulate the discussion of the economic use of phytoestrogens in therapy instead of the known animal and synthetic estrogens. This point waits the discovery of a phytoestrogen which is enough potent biologically.

15 E. Bottlglioni and R. L. Sturani, Arch. E. Moragl. Pathologia [Genova] 4, 1149 [1949].

16 B. Gaudlitz, Inaug. Diss., Berlin 1955.

17 R. Moberg, Thesis, Stockholm 1955.

18 Th. Dougherty and J. A. Frank, J. Lab. clin. Med. 42, 530 [1953].

\begin{tabular}{|c|c|c|c|}
\hline \multirow{2}{*}{ Indicator } & \multirow{2}{*}{ Control } & \multicolumn{2}{|c|}{$\beta$-sitosterol } \\
\hline & & $1 \mu \mathrm{g}$. & $50 \mu \mathrm{g}$. \\
\hline $\begin{array}{l}\text { Av. body weight } \\
\text { (gm.) }\end{array}$ & $9.80 \pm 0.22$ & $10.38 \pm 0.33 *$ & $9.37 \pm 0.41^{*}$ \\
\hline $\begin{array}{l}\text { Av. Uterine wt. } \\
(\mathrm{mg} . \%)\end{array}$ & $54.50 \pm 2.11$ & $68.24 \pm 3$ & $58.61 \pm 2.49 *$ \\
\hline $\begin{array}{l}\text { R.B.Cs. (mil./ } \\
\text { cu. mm.) }\end{array}$ & $5.51 \pm 0.19$ & $4.61 \pm 0.18$ & $3.49 \pm 0.23$ \\
\hline $\begin{array}{l}\text { ml.) } \\
\text { (1). }\end{array}$ & $12.03 \pm 0.57$ & $10.19 \pm 0.12$ & $10.56 \pm 0.18$ \\
\hline PCV (mm.) & $36.11 \pm 0.37$ & $30.65 \pm 0.49$ & $31.76 \pm 0.49$ \\
\hline $\begin{array}{l}\text { Sed. rate (mm./ } \\
3 \text { hours) }\end{array}$ & $0.84 \pm 0.16$ & $1.11 \pm 0.08 *$ & $0.70 \pm 0.13 *$ \\
\hline $\begin{array}{l}\text { W.B.Cs.(thous.) } \\
\text { cu. mm.) }\end{array}$ & $13.03 \pm 0.48$ & $17.22 \pm 0.47$ & $18.65 \pm 0.63$ \\
\hline Stab [\%] & $0.53 \pm 0.09$ & $2.15 \pm 0.30$ & $3.16 \pm 0.26$ \\
\hline Neutrophils [\%] & $15.42 \pm 0.64$ & $39.88+1.92$ & $42.12+1.83$ \\
\hline Eosinophils [\%] & $2.00 \pm 0.20$ & $0.65 \pm 0.15$ & $1.00 \pm 0.22$ \\
\hline Basophils [\%] & $0.23 \pm 0.09$ & $0.06 \pm 0.04 *$ & - \\
\hline $\begin{array}{l}\text { Lymphocytes } \\
{[\%]}\end{array}$ & $79.70 \pm 0.78$ & $58.34 \pm 2.06$ & $50.52 \pm 1.91$ \\
\hline Monocytes [\%] & $2.12 \pm 0.17$ & $2.98 \pm 0.37$ & $3.20 \pm 0.21$ \\
\hline
\end{tabular}

Table 1. Blood changes in female mice after administration of $1 \mu \mathrm{g}$. and $50 \mu \mathrm{g}$. of $\beta$-sitosterol. $\pm=$ Standard error. * = The difference between control and experimental mice was insignificant. This difference in all the other data is statistically significant.

\section{Photoperiodische Steuerung der 15-tägigen lunaren Metamorphose-Periodik von Clunio-Populationen (Diptera: Chironomidae)}

\section{Dietrich NeumanN \\ Zoologisches Institut der Universität Würzburg \\ (Z. Naturforschg. 20 b, 818-819 [1965]; eingegangen am 17. Mai 1965)}

Die an gezeitenstarken Meeresküsten lebenden Populationen der Mücke Clunio marinus zeigen eine annähernd 15-tägige Schlüpf- und FortpflanzungsPeriodik ihrer Imagines ${ }^{1-3}$. Die Schlüpfphasen sind auf bestimmte Niedrigwasserzeiten des 14,8-tägigen, zwischen Spring- und Nipptiden wechselnden Gezeitenzyklus abgestimmt und laufen damit parallel zum 29,5tägigen Mondphasenzyklus. An der Normandieküste (Frankreich) beginnen sie an den Voll- und Neumondterminen und erstrecken sich jeweils über 4-5 Tage, an denen die nur wenige Stdn. lebenden Imagines um die Zeit des Nachmittags-Niedrigwassers erscheinen.

Wenn Clunio-Kulturen im künstlichen Tag-NachtWechsel gehalten werden, so tritt keine periodische Schlüpfverteilung auf (Abb. 1 a, Summendiagramm von 5 Kulturen); die Entwicklungsdauer schwankt zwischen 45 und etwa 100 Tagen. Wenn die Kulturen zusätzlich einem künstlichen Mondlicht ausgesetzt werden, und zwar in einem 30-tägigen Belichtungszyklus (entsprechend der Dauer eines synodischen Monats),

1 R. Chevrel, Arch. Zool. exp. gen. Ser. 3, 2, 583 [1894].

2 H. Caspers, Arch. Hydrobiol., Suppl. Bd. 18, 415 [1951]. so stellt sich eine auffällige 15-tägige Schlüpfperiodik ein (Abb. 1 b, Summendiagramm von 4 Kulturen, Schlüpfmaxima bei $63,1,78,3$ und 92,8 Tagen). Die Schlüpfphasen lassen sich durch eine Verschiebung des künstlichen Mondlichtzyklus auch auf andere Tage verlegen, sie fallen jedoch stets auf die Tage mit Dämmerlichtnacht und auf die zwei Wochen später liegengen Tage. Diese im Laboratorium ausgelöste Periodik entspricht der Schlüpfperiodik der Freilandpopulationen. Vermutlich können daher auch im Freiland die Schlüpfphasen unabhängig von den Gezeitenbedingungen allein durch den periodischen Wechsel von mondhellen und dunklen Nächten gesteuert werden.

Die Metamorphosestadien treten jeweils erst kurz vor den Schlüpftagen auf (Puppendauer + : $2-3$, ô: $3-5$ Tage bei $20^{\circ} \mathrm{C}$ ). Die 15-tägige Schlüpfperiodik beruht daher auf einer MetamorphosePeriodik der Populationen. Erhält eine Larvenpopulation im IV. Larvenstadium nur eine einmalige Dämmerlichtbehandlung, aus der sie weder einen Hinweis auf eine 30-tägige noch eine 15-tägige Periodendauer entnehmen kann, so stellt sich gleichfalls eine annähernd zweiwöchige Periodik ein (Abb. l c, Summendiagramm von 6 Kulturen aus 3 Versuchsserien, Schlüpfmaxima bei $62,0,76,2$ und 89,0 Tagen). Die Metamorphose-Periodik der Larvenpopulationen muß daher auf einer endogenen, und zwar angeborenen Periodik beruhen, die mit gleicher Periodendauer in

3 Н. Ока и. Н. НаSнiмото, Biol. Zbl. 78, 545 [1959]. 


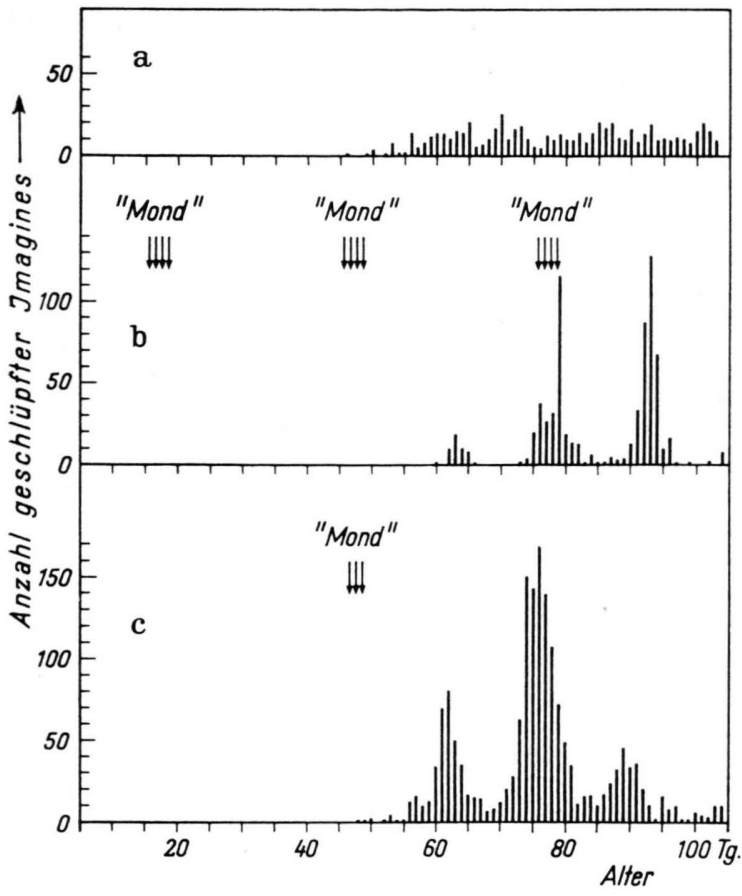

Abb. 1. Clunio marinus (Normandiepopulation). Schlüpfverteilung der Imagines in Abhängigkeit von den photoperiodischen Aufzuchtbedingungen der Larven. a-c: Täglicher Licht-Dunkel-Wechsel mit 16-stdg. Licht- und 8-stdg. Dunkelzeit (Leuchtstoffröhren, ca. $400 \mathrm{Lux} ; 20^{\circ} \mathrm{C}$ ). a) ohne Dämmerlicht während der Dunkelzeit, b) mit Dämmerlicht von etwa 0,3 Lux („Mond“), alle 30 Tage in jeweils 4 aufeinanderfolgenden Nächten. c) mit einmaliger Dämmerlichtbehandlung (0,3 Lux in 3 aufeinanderfolgenden Nächten).

4 D. Neumann, Verh. dtsch. zool. Ges. 1962, 275.

5 E. Bünning u. D. Müller, Z. Naturforschg. 16 b, 391 [1961]. jeder Larve läuft und die Metamorphose der ausgewachsenen Larven nur alle 2 Wochen zuläßt. Das „Mondlicht" wirkt als Zeitgeber, welcher die endogene Periodik der Larven synchronisiert. Die endogene Periodendauer ist möglicherweise etwas kürzer als die eingesteuerte (in Abb. 1 c: 13 - 14 Tage).

Die gleichen Befunde ergaben sich auch bei ClunioPopulationen von der spanischen Atlantikküste und der Mittelmeerküste. Bei einer Nordsee-Population (Helgoland) war unter den gleichen Versuchsbedingungen keine prägnante Periodik auszulösen; der Zeitgeber für diese Population wird noch untersucht. Die genaue zeitliche Abstimmung der Schlüpfphasen auf die alle 15 Tage in den gleichen Tageszeitbereich fallenden Niedrigwasserzeiten wird durch die Kombinierung der 15-tägigen Metamorphose-Periodik mit einem tageszeitlich gebundenen Schlüpfen gewährleistet ${ }^{4}$.

Die Versuche belegen erstmals bei einer Tierart die photoperiodische Steuerung einer 15-tägigen lunaren Periodik. Mit Hilfe des Mondlichtes ließen sich auch die 15-tägige Fortpflanzungs-Periodik der Braunalge Dictyota $^{5}$, die 30-tägige lunare Schwärmperiodik des Polychaeten Platynereis ${ }^{6}$ und die 30 -tägige lunare Metaphormose-Periodik einer Clunio-Population ${ }^{4}$ induzieren. Die geringe Beleuchtungsstärke des Mondlichtes kann folglich sowohl zweiwöchige als auch vierwöchige Periodizitäts-Erscheinungen von Organismen der Gezeitenzone steuern.

Die Untersuchungen werden an anderer Stelle ausführlicher veröffentlicht.

Mit Unterstützung durch die De u t s che Fors chung s g e m e in s c h a f t.

6 C. Hauenschild, Naturwissenschaften 43, 361 [1956].

\section{Die Hemmung des Phytochrom-induzierten Kotyledonenwachstums durch Actinomycin D}

\section{Hans Mohr, Inge Schlickewei und Herbert Lange}

Botanisches Institut der Universität Freiburg i. Br.

(Z. Naturforschg. 20 b, 819-821 [1965]; eingegangen am 2. Juni 1965)

Die Regulation der pflanzlichen Morphogenese durch Licht („Photomorphogenese“) erfolgt bekanntlich über das neuerdings "Phytochrom“ genannte Reversible Hellrot-Dunkelrot Pigmentsystem ${ }^{1}$. Die morphogenetische Wirkung des Phytochroms beruht auf der photochemischen Bildung des physiologisch aktiven Phytochroms $730\left(=\mathrm{P}_{730}\right)$. Während die komplizierte Photo-

1 H. Moнr, Naturwiss. Rdsch. 18, 101 [1965].

2 B. Hock u. H. MoHr, Planta 61, 209 [1964].

3 H. Mohr u. H. Lange, Naturwissenschaften 52, 261 [1965].

4 W. L. Butler, S. B. Hendricks u. H. W. Siegelman, in: Chemistry and Biochemistry of Plant Pigments; T. W. Goodwin, edit.; Acad. Press New York - London [1965]. chemie des Phytochroms sowohl in vivo als auch in vitro in jüngerer Zeit genau untersucht werden konnte ${ }^{4}$, besteht hinsichtlich der Wirkungsweise des physiologisch aktiven $\mathrm{P}_{730}$ trotz vieler diesbezüglicher Untersuchungen noch keine einheitliche Auffassung ${ }^{1,5}$.

Wir haben kürzlich die Hypothese ausführlich begründet ${ }^{1,2}$, daß die Wirkung des Phytochroms im Rahmen der Photomorphogenese von Keimpflanzen auf eine differentielle Genaktivierung durch $\mathrm{P}_{730}$ zurückzuführen sei. Nach dieser Auffassung soll $\mathrm{P}_{730}$ über eine „Signalkette“ potentiell aktive Gene aktivieren. Welche Gene in einer bestimmten Zelle des vielzelligen Systems aktivierbar („potentiell aktiv“) sind, hängt vom jeweiligen Differenzierungszustand dieser Zelle ab.

5 S. B. Hendricks u. H. A. Borthwick, in: Chemistry and Biochemistry of Plant Pigments; T. W. Goodwin, edit.; Acad. Press New York - London [1965]. 